\title{
Numerical Simulations of the Flame of a Single Coaxial Injector
}

\author{
Victor P. Zhukov and Markus Feil \\ Institute of Space Propulsion, German Aerospace Center (DLR), Langer Grund, 74239 Hardthausen, Germany \\ Correspondence should be addressed to Victor P. Zhukov; victor.zhukov@dlr.de
}

Received 19 January 2017; Accepted 8 May 2017; Published 27 June 2017

Academic Editor: Corin Segal

Copyright (C) 2017 Victor P. Zhukov and Markus Feil. This is an open access article distributed under the Creative Commons Attribution License, which permits unrestricted use, distribution, and reproduction in any medium, provided the original work is properly cited.

\begin{abstract}
The processes of mixing and combustion in the jet of a shear-coaxial injector are investigated. Two test cases (nonreacting and reacting) are simulated using the commercial computational fluid dynamics code ANSYS CFX. The first test case is an experiment on the mixing in a nonreacting coaxial jet carried out with the use of planar laser induced fluorescence (PLIF). The second test case is an experiment on the visualization of hydrogen-oxygen flame using PLIF of $\mathrm{OH}$ in a single injector combustion chamber at pressure of 53 bar. In the first test case, the two-dimensional axisymmetric simulations are performed using the shear-stress turbulence (SST) model. Due to the dominant flow unsteadiness in the second test case, the turbulence is modeled using transient SAS (Scale-Adaptive Simulation) model. The combustion is modeled using the burning velocity model (BVM) while both two- and three-dimensional simulations are carried out. The numerical model agrees with the experimental data very well in the first test case and adequately in the second test case.
\end{abstract}

\section{Introduction}

Shear-coaxial injectors have a wide usage in rocketry. They are used practically in all hydrogen rocket engines. Though operated engines use multi-injector systems which consist of hundreds of coaxial injectors, the flame of even a single injector is an actual problem which is not fully understood yet.

Tucker et al. [1] started a cooperation between several institutes to compare available results of numerical simulations on a standard case, contrasting several methods with each other and with experimental data. The test case was a research combustion chamber at the Pennsylvania State University (PSU). The heat flux and wall temperature measurements by Pal et al. [2] were used as the benchmark experimental data. In the experiment, a gaseous-gaseous coaxial injector with similar dimensions as the SSME (Space Shuttle Main Engine) injector was tested inside an axisymmetric combustion chamber. In the cooperation project, Reynolds Averaged Navier-Stokes (RANS), an Unsteady Reynolds Averaged Navier-Stokes (URANS), and three different Large Eddy Simulations (LES) approaches were applied. Different meshes with sizes between 0.25 and 255 million cells were used. The chemical reaction of the propellants was modeled using detailed chemical kinetics. The RANS simulation was the simplest among the performed simulations with respect to the required computational resources and turbulence modeling. However, RANS is a technique not yet outdated and is used successfully by many researchers for the simulation of reacting flows in rocket combustion chambers. Thus, later, Zhukov [3] showed that RANS is able to give a good prediction of the wall heat fluxes in the test case of Pal et al. [2]. Unfortunately, there is a big uncertainty about error sources, like mixing, heat transfer, and combustion characteristics in experiment [2] that does not allow qualifying a method as incorrect in the case of disagreement with experimental data. It was shown by Tucker et al. [1] that the results of the various codes are different with an underprediction of mixing by the RANS simulation and an overprediction of mixing with the LES simulations. The two-dimensional axisymmetric RANS shows higher flux at the combustion chamber walls than the experiment, while the LES simulations show lower wall heat fluxes. The threedimensional simulations showed a smoother heat flux curve than two-dimensional simulations. The two-dimensional axisymmetric nature of RANS simulations implies difficulties 
in the mixing of hydrogen and oxygen at the centreline, hence resulting in a thin flame sheet, while unsteady simulations show a much more realistic representation of the flow.

At present, there is no commonly used method for the computational fluid dynamics (CFD) modeling of rocket combustion chambers. It is understood that we need new and better models of turbulence and turbulent combustion, and this work is carried out by many researchers. However, there is also the problem of the usage of already existing CFD methods for the simulations of rocket combustion chambers. For instance, the authors of the large cooperative work on CFD modeling of rocket combustion chambers could not explain their results, namely, why some simulations show higher wall heat fluxes than others [1]. The goal of the present work is to simulate the flame of single coaxial injector using existing turbulence and combustion models and thus to evaluate their performance and limitations.

Oefelein [4] carried out simulations of mixing and combustion in oxygen-hydrogen coaxial jet flames. The simulations were performed using both the LES and DirectNumerical-Simulation (DNS) techniques employing detailed treatments of thermodynamics, transport, and chemical kinetics. The modeled combustion chamber had a single coaxial injector. Cryogenic hydrogen and oxygen were injected at a supercritical pressure. The study is focused exclusively on the near-field region just downstream of the injector. The detailed numerical analysis of the flow in the wake of the injector post gave an idea about the flame holding mechanism which is provided by strong counter-recirculating vortices establishing a zone of hot fuel-rich products in the vicinity of the injector tip. The flame development within the stagnation region is dominated by diffusion and enhanced by convective energy transport from the backflow of fuel-rich combustion products. The backflow of hot products both sustains the flame and promotes the jet breakup of the cold oxygen core.

Many research groups focus their research on simulations of flames with liquid oxygen $\left(\mathrm{H}_{2} / \mathrm{LOx}\right.$ or $\left.\mathrm{CH}_{4} / \mathrm{LOx}\right)$. These works are off the scope of the current work and are not considered here. Of course, the case of combustion of cryogenic propellants is more relevant to application as the gas-gas case, but it is difficult to obtain experimental data which is suitable for validation purposes. The case of gas-gas combustion has already complicated physics, so it is sensible to begin the development of the numerical model of the flow in a rocket combustion chamber from this test case rather than from the more complicated LOx case. There is also another problem associated with the LOx case. Experiments with cryogenic propellants are more difficult and more expensive; as a consequence diagnostics suffer in such experiments. For example, most of the researchers, who work on modeling of cryogenic rocket combustion chambers, use the "Mascotte" test case [5] for validation. However, the experiment has provided the information only about the middle field of the flame. Parameter such as flame length, by which a numerical model can be assessed easily, is not available in [5]. By this means, "Mascotte" test case is practically not suitable for the validation of a numerical model concerned with flame development. There is no detailed information about the flame front and about the vortex shedding and development of the Kelvin-Helmholtz instability in the wake of the injector post available, which is important for turbulence and turbulent combustion modeling.

In the current work we have used experimental data by Schumaker [6] and by Vaidyanathan [7] for the validation of our numerical model. The experimental data used here were obtained with the use of planar laser induced fluorescence (PLIF). The experiments in $[6,7]$ show more information about processes in coaxial jets than the "Mascotte" test case [5]; however, they were carried out at injection temperature of $300 \mathrm{~K}$ which is limiting the validity range of the tested CFD model.

\section{Modeling of Mixing in the Flow of a Coaxial Injector}

In order to react chemically, propellants must mix with each other at a molecular level. Rocket combustion chambers are subject to high pressures and temperatures; the timescales of chemical reactions are smaller than the ones of mixing. The rate of combustion is determined by the mixing which is therefore the limiting stage. Hence, the accurate modeling of the mixing processes is of great importance.

2.1. Experiments. The mixing of gases injected by a shearcoaxial injector was examined by Schumaker [6] in a combustion chamber at the University of Michigan. The attention was turned to the length of the inner core in dependency of velocity and density ratios. The experimental setup consisted of the rectangular shaped combustion chamber $(50.8 \mathrm{~mm} \times$ $50.8 \mathrm{~mm} \times 266.7 \mathrm{~mm}$ ) with a single shear-coaxial injector. The measurements were taken at ambient conditions.

Helium and air (seeded with acetone) were used as fluids. The seeded acetone enabled the quantitative measurements of the mass fractions using PLIF in the inner jet. The experimental uncertainty amounted up to $8.2 \%$ due to the complex measurements of the molar fraction of the inner jet fluid based on the acetone. The quantitative data was achieved using a second window, which was placed before the entry into the chamber to have a reference signal for the inner jet acetone concentration.

The main objective of these measurements was to obtain the core length of the inner jet, described by the position where the air acetone mixture mass fraction equals 0.89 . Mixture fraction can be defined by different ways depending on the specific case. For hydrogen-oxygen combustion chambers, mixture (mass) fraction is normally defined as the total mass fraction of the hydrogen element in the mixture. However, in the study by Schumaker, mixture fraction is the mass fraction of oxidizer in the mixture because the oxidizer was traced using PLIF but not "fuel." The value of 0.89 was chosen to be equivalent to the stoichiometric composition in the more interesting case of $\mathrm{H}_{2}-\mathrm{O}_{2}$ mixing. The distribution of the mixture fraction along the axis of the chamber and also the mixture fraction distribution along the radial direction were used to validate the numerical simulations concerning 
TABLE 1: Injection conditions for the nonreacting ("mixing") test case [6].

\begin{tabular}{|c|c|c|c|c|c|c|}
\hline Parameter & Case $\mathrm{C} 1$ & Case C2 & Case C3 & Case C4 & Case C5 & Case C6 \\
\hline Velocity $V_{\text {fuel }}, \mathrm{m} / \mathrm{s}$ & 64.5 & 59.3 & 54.7 & 47.6 & 42.2 & 38.2 \\
\hline Velocity $V_{\text {oxid }}, \mathrm{m} / \mathrm{s}$ & 6.25 & 11.4 & 17.1 & 24.8 & 31.0 & 35.8 \\
\hline Helium mass flux $\phi_{\text {fuel }}, \mathrm{g} / \mathrm{s}$ & 0.061 & 0.251 & 0.232 & 0.202 & 0.302 & 0.347 \\
\hline Air mass flux $\phi_{\text {fuel }}, \mathrm{g} / \mathrm{s}$ & 0.273 & 0.110 & 0.167 & 0.242 & 0.179 & 0.162 \\
\hline
\end{tabular}

TABLE 2: Experimental boundary conditions $[6,7]$.

\begin{tabular}{lcc}
\hline Parameter & Nonreacting test case [6] & Reacting test case [7] \\
\hline Oxidizer injector $d_{i}$ & $3 \mathrm{~mm}$ & $1.2 \mathrm{~mm}$ \\
Injector tip $l$ & $0.89 \mathrm{~mm}$ & $0.5 \mathrm{~mm}$ \\
Oxidizer injector $d_{e}$ & 7.52 & $2.2 \mathrm{~mm}$ \\
Temperature & $300 \mathrm{~K}$ & $300 \mathrm{~K}$ \\
Pressure & $0.961 \mathrm{bar}$ & $53 \mathrm{bar}$ \\
Momentum flux ratio $J$ & $0.13-12$ & 0.4 \\
\hline
\end{tabular}

the mixing behavior. For present work, the most important test case is case $\mathrm{C} 4$ with a momentum flux ratio $J$,

$$
J=\frac{\rho_{\text {fuel }} V_{\text {fuel }}^{2}}{\rho_{\text {oxid }} V_{\text {oxid }}^{2}}=0.42,
$$

and velocity ratio $K$,

$$
K=\frac{V_{\text {fuel }}}{V_{\text {oxid }}}=0.52 \text {, }
$$

which are the closest case to the following combustion modeling test case [7] $(J=0.4, K=0.4)$. The second in importance are cases $\mathrm{C} 1-\mathrm{C} 3$ which are closer to rocket coaxial injectors by $J$ number. The overview of the conditions of the experiments is given in Tables 1 and 2 .

In the considered experiments of Schumaker [6] and Vaidyanathan [7], the chambers were equipped with a singleelement shear-coaxial injector. Shear-coaxial injectors are very common for hydrogen rocket engines. In both simulated experiments $[6,7]$, the injector was located on the longitudinal chamber axis. The injectors $[6,7]$ have a typical design for shear-coaxial injectors. "Oxidizer" was fed through a tube positioned at the center of the injector. "Fuel" was injected through an annular gap surrounding the oxidizer injector. The tube separating "fuel" and "oxidizer" (oxidizer post) ended by an injector tip which was rectangular in cross section and did not protrude and was not recessed relative to the material surrounding the injector, that is, without "tapering" and "recess".

2.2. Simulations. The simulations have been performed using the commercial computational fluid dynamics code ANSYS CFX [8], which utilizes the finite volume element method (FVEM). The numerical simulations of the flow inside the chamber have been carried out in a $2 \mathrm{D}$ (2-dimensional) axisymmetric computational domain. The domain includes the injectors, but not the injector dome. In the numerical domain the turbulent flow of a compressible fluid has been simulated.
The simulations were performed on hexahedral structured meshes which are clustered near the walls. The numerical meshes were generated using the computer program ICEM from package ANSYS CFD. Six meshes with different resolutions were tested until the final mesh, which provides a mesh independent solution with a reasonable amount of nodes, has been found. During the mesh convergence study the spacing was varied from 2 nodes at the injector post to 58 nodes. The final mesh consists of 112 thousands nodes with 29 nodes at the injector tip $(l=0.89 \mathrm{~mm}$, see Table 2$)$. The mesh is refined near the injector post and the injector walls. The expansion ratio next to the walls was set to 1.2 , resulting in a $y^{+}$resolution smaller than 1 , where $y^{+}$is the dimensionless distance from the wall [8].

The flow in the combustion chamber has been modeled as a stationary solution of Reynolds Averaged Navier-Stokes (RANS) equations. The turbulence was modeled with the help of the Shear-Stress-Transport (SST) model [8] using the standard values of the coefficients and the "automatic" wall treatment. The transport has been modeled with a turbulent Schmidt number of 0.7 (the value of 0.7 is recommended for high-Reynolds-number jet flows by Yimer et al. [9]). The turbulent Prandtl number was set to the default value in CFX which equals 0.9 . Fluid media have been modeled as an ideal mixture of ideal gases. Accordingly, the mixture components have properties of ideal gases at ambient conditions. In the reacting test case, it is necessary to take into account the change of thermodynamic properties with temperature. The model of thermodynamic properties, which was used for the "combustion" test case, will be described later in the corresponding section.

Usually, the details of experiments are not sufficient to establish clearly the boundary conditions, namely, the level of turbulence at inlets and thermal conditions at walls. Hence, the comparison with experimental data was used to define the missing boundary conditions.

2.3. Results and Discussion. The nonreacting experiments with focus on mixing [6] have been used for the verification 


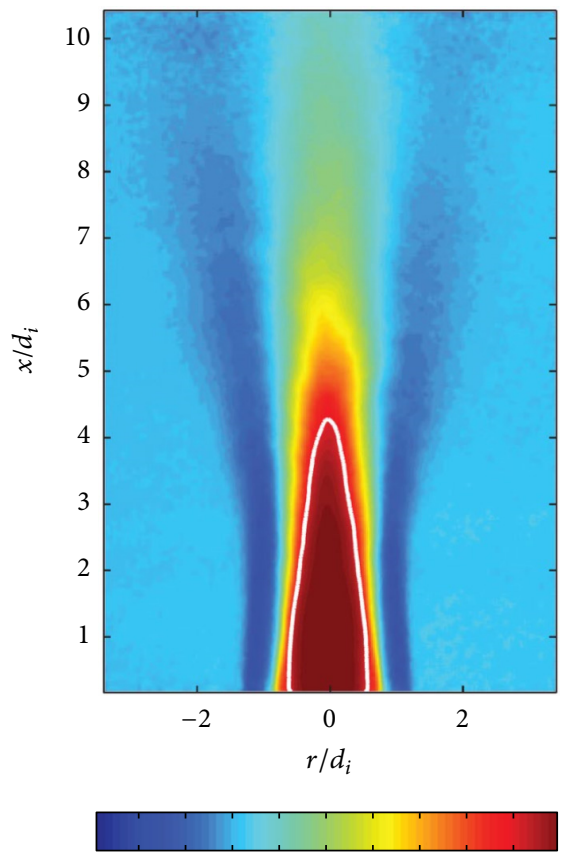

$\begin{array}{lllllllll}0.1 & 0.2 & 0.3 & 0.4 & 0.5 & 0.6 & 0.7 & 0.8 & 0.9\end{array}$

(a)

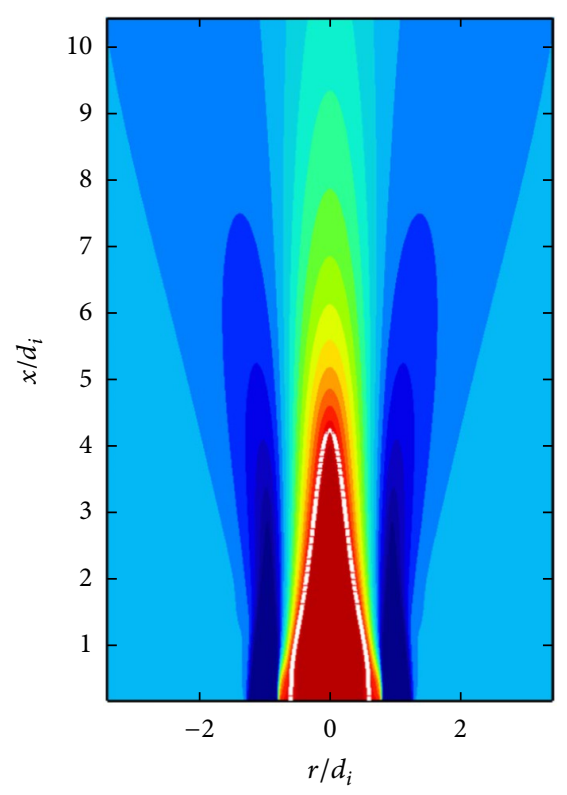

$\begin{array}{lllllllllll}0.0 & 0.1 & 0.2 & 0.3 & 0.4 & 0.5 & 0.6 & 0.7 & 0.8 & 0.9 & 1.0\end{array}$

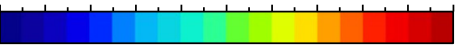

(b)

FIGURE 1: Mass fraction distribution for case C2, (a)-experimental data [6], (b) - simulations (the white line corresponds to the mixture fraction of 0.89 ).

and validation of the numerical model. The flow inside the chamber is rather simple in this experiment, and even the coarsest mesh gives acceptable results. One of the goals of the present study was to assess the impact of the spacial discretization on the simulating results. To study the effect of mesh on simulating results, the results obtained on different meshes have been compared with each other and with experiment [6]. An example of comparison with the experiment is shown in Figure 1. The most convenient parameter for the comparison with the experiment is the mixing length in the present case because it is possible to assess the accuracy of simulation by one single parameter. (In the present work, the mixing length is defined as the distance, at which the oxidizer mixture fraction reaches 0.89 on the chamber axis.) Other parameters such as the thickness of the central jet are not so informative as mixing length because it depends mainly on the diameter of the injector but not on the numerical model; see Figure 1.

The comparison of simulating results obtained on different meshes shows that the simulated mixing length is not very sensitive to mesh density; however, the order of the grid convergence is very low and amounts to around 0.5; see Figure 2. The spacing at the injector tip or the number of cells there was used as a measure of mesh density. As we can see in Figure 2, all points except one lie on one line which has the inclination of about 0.5 . The point out of the line corresponds to the very coarse mesh which has only two cells at the injector tip; nevertheless, this mesh provides results comparable to the experimental data. The errors on the graph were calculated using Richardson extrapolation with

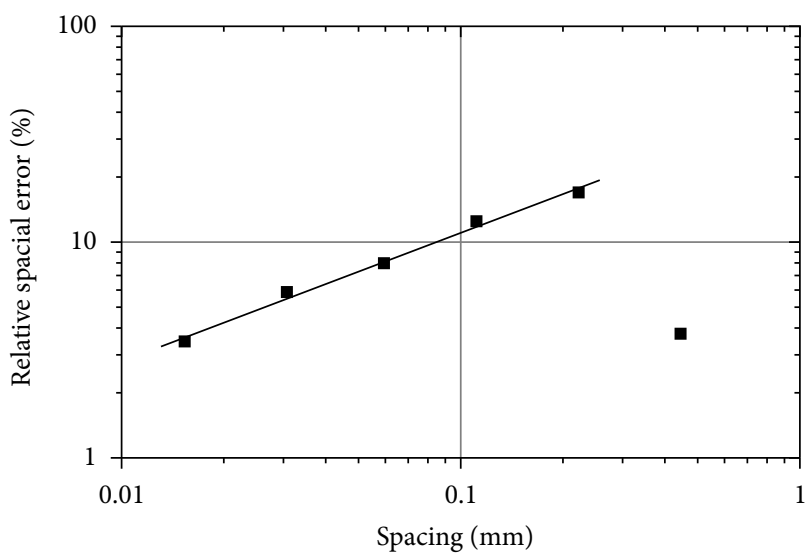

FIGURE 2: Error in the mixing length as the mesh is refined at the oxygen post, case $\mathrm{C} 4$.

the order of convergence of 0.5 . The governing equations are discretized using a second-order scheme; however, they are not solved relative to the mixing length but relative to other variables: $\rho, v, P$, and so on. Such integral parameters as mixing length depend on the governing variables but generally nonlinearly.

While the mixing length weakly depends on mesh density, the flow around the injector tip is very sensitive to mesh density. The zone right behind the oxygen post plays an important role because it is responsible for the flame holding. If we are not interested in the processes on the flame front 


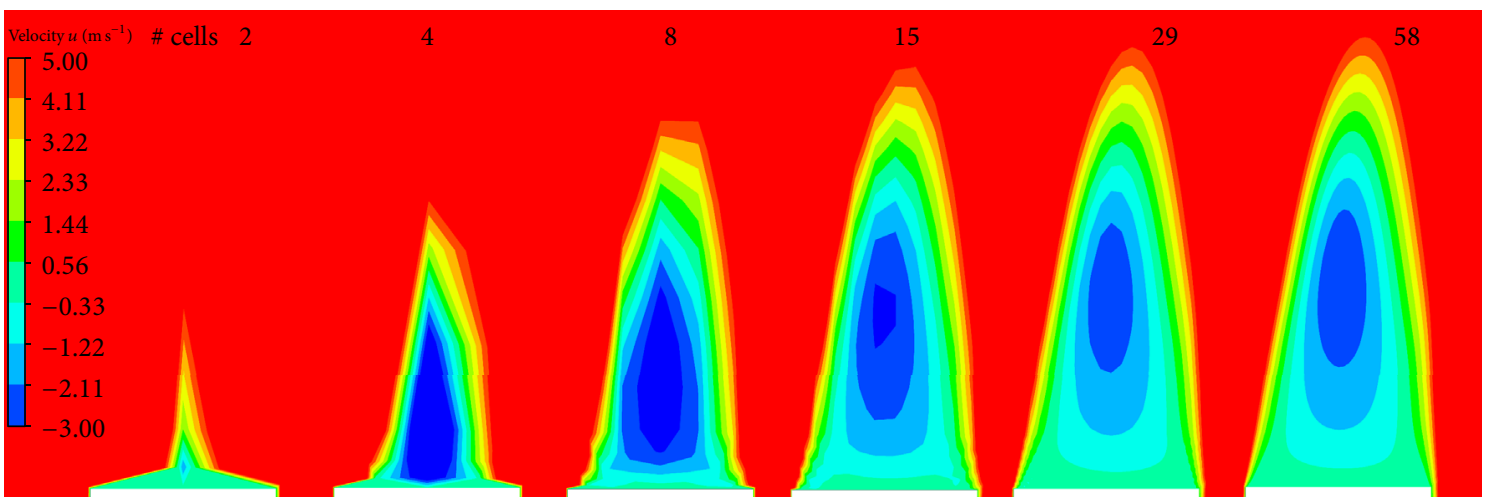

FIGURE 3: The change of the velocity field near the injector tip with the increase of the mesh density from 2 cells at the injector tip to 58 cells, case C4. (Red corresponds to axial velocities above $5 \mathrm{~m} / \mathrm{s}$; blue corresponds to axial velocities below $-3 \mathrm{~m} / \mathrm{s}$.)

and at the injector tip, it is possible to get acceptable results on a relatively coarse mesh. However, this requires that the model will be able to provide flame holding and stabilization behind the injector tip; otherwise, the flame will be blown out from combustion chamber in simulations. As we can see in Figure 3, the formation of the recirculation zone needed for flame holding requires a mesh with 9 nodes at least at the injector tip.

It could be thought that the inclusion of the results obtained on mesh with only two cells on the injector tip in Figure 3 has no sense because this mesh is obviously too rough. However, if we take into account the fact that simulations of heat loads in combustion chambers with multielement injector require three-dimensional (3D) modeling [10], we will see reasons that make such comparison sensible. Meshes, which are used for the modeling of rocket combustion chambers, are very large in spite of very poor mesh resolution. For instance, a test case with a calorimetric subscale combustion chamber is known, presented at the 3rd international workshop "Rocket Combustion Modeling" [11]. All participants of the workshop used meshes with a resolution of only several cells at the injector tip for this combustion chamber [12-14]. The use of meshes with poor resolution for modeling of rocket combustion chambers is a compromise between the accuracy of simulations and available computational resources. Therefore, the comparison with the results of very rough meshes is of some methodological interest.

In coaxial injectors, fuel flows within a narrow gap, which amounts to $1.37 \mathrm{~mm}$ in our case; see Table 2. In such conditions, the laminarization of the flow occurs due to the viscous losses at the walls. To account for this effect, the laminar-transition "Gamma-Theta" model [15], which is built in CFX, has been used. The two additional transport equations have been employed, one for the intermittency and one for the transition onset criteria.

The comparison with the experiment was performed not only by mixing length but also by axial profiles of oxidizer mass fraction. The distribution of oxidizer mass fraction along the axis of the injector is shown for case C3 in Figure 4. As we can see in Figures 1 and 4, the numerical

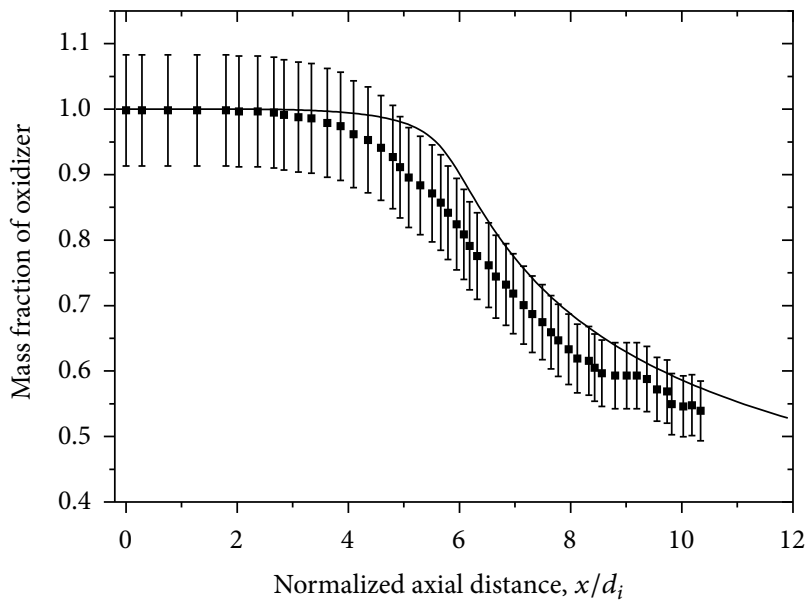

FIgURE 4: Mass fraction of the oxidizer at the axis of the injector (symbols-experimental data [6]; line-simulation, case C3).

results agree well with the experimental data. The present results were obtained on the mesh with 29 cells at the injector tip. In Figure 1, the saturated red shows the central jet of pure "oxidizer" (air); the saturated blue denotes pure "fuel" (helium). In both axial and radial directions, the numerical model gives the distribution of oxygen which is very close to the observed distribution in the experiments. (It is necessary to note that the experimental results and the simulations have slightly different color maps in Figure 1.) The comparison of the simulations for all the six cases shows a good agreement with the experiments. For some cases, the mixing is slightly overpredicted; for others it is slightly underpredicted. The simulations have been performed for all cases, C1-C6 (see Table 1), and the numerical model agrees with the experimental mixing length within the measurement accuracy for all cases. In order not to overload the text with redundant information, only the most relevant and typical results have been shown here.

In rocket engines, propellants are injected at speeds of tens to hundreds of meters per second which is too high even for hydrogen-oxygen flames. Under such conditions, the 


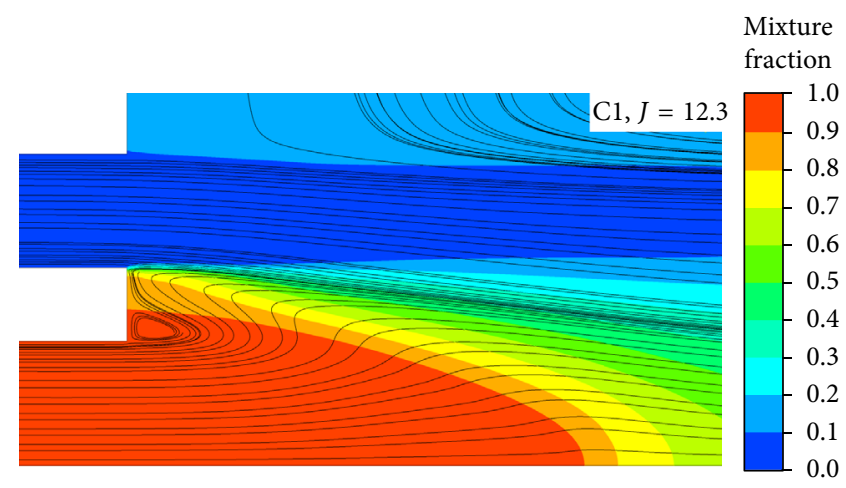

(a)

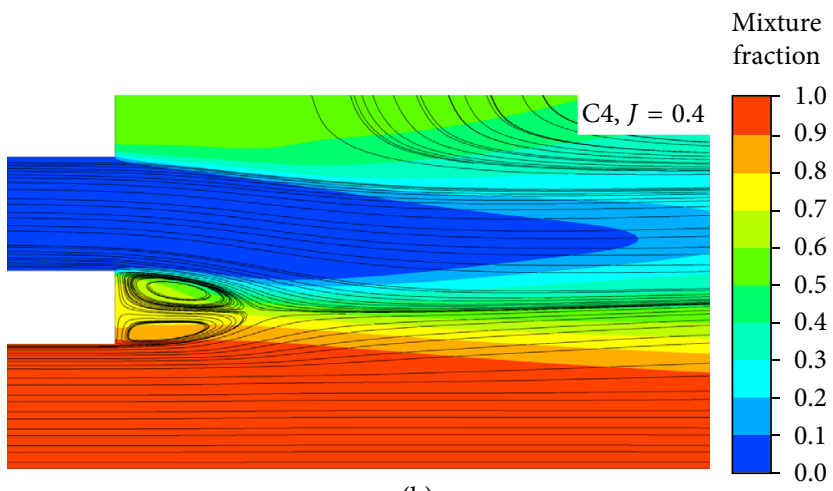

(b)

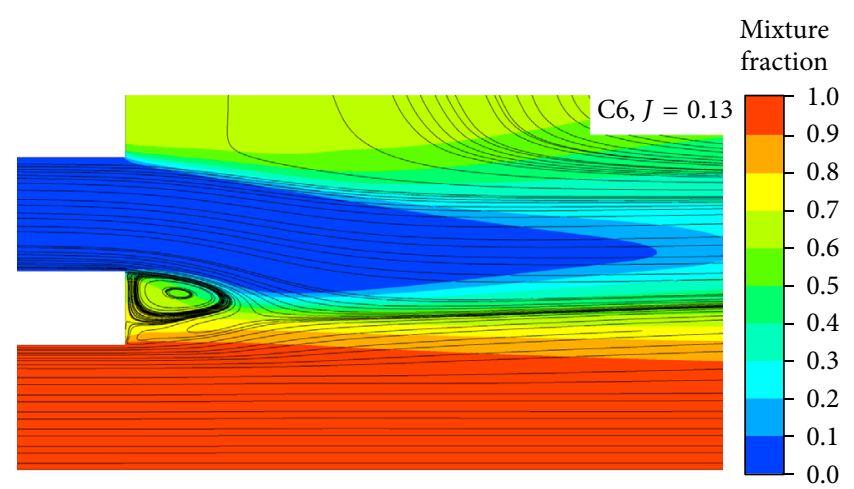

(c)

FIGURE 5: Flow in the wake of injector post at different momentum flux ratios. Blue color corresponds to helium (fuel) and red to air (oxidizer).

flame can be sustained only when the initial point of the flame is located in the stagnation area behind the oxygen post. The average flow field near the injector post obtained using the simulations is shown for several test cases in Figure 5. In the figure, we see the recirculation of gas behind the injector post. The recirculation provides the mixing of reactants and the residence time sufficient for the formation of initial flame kernel. The recirculation is formed at the injector post edge that is next to the flow with the less momentum. If the $J$ number (the ratio of momentums) is close to unity, then two vortices are formed at the both edges of the injector post; see case C4 in Figure 5. The amount of gas involved in the recirculation reaches a maximum near the $J$ number of unity. Nevertheless, the $J$ number of unity is not optimal for the flame holding. The optimum for the flame holding is reached near the $J$ number of 10 , when the gas mixture with the mixture fraction of 0.89 (i.e., the stoichiometric mixture of $\mathrm{H}_{2}$ and $\mathrm{O}_{2}$ ) is involved in the recirculation. The $J$ number also affects the atomization of the central jet of oxidizer: the length of oxygen jet declines in coaxial flows with $J^{-0.5}$ at supercritical conditions [16]. As we can see, the larger $J$ numbers are also preferable from the point of view of flame length.

\section{Simulations of the Flame of a Single Coaxial Injector}

The ultimate goal of the current work is a numerical model which is able to simulate reactive flows in a rocket combustion chamber. In this case, the CFD model should be validated not only against the experiments with inert gases, but also against combustion experiments. The flame of a shear-coaxial injector is a sufficiently complex object and incorporates all features of a flame. Hence, to simulate the flame of a shear-coaxial injector it is necessary to model all properties of the flame. Therefore, the model can be validated comprehensively by the experiments on the flame of a single shear-coaxial injector. Unfortunately, more complicated CFD models require more boundary conditions and more experimental data for their validation; therefore, the corresponding experiments are complicated and rare.

3.1. Experiments. Vaidyanathan [7] carried out the observations of the near field of the flame of a shear-coaxial injector. In the experiments, the distribution of $\mathrm{OH}$ radicals was measured using PLIF. The chamber is made of copper and has a rectangular shape with a $25.4 \mathrm{~mm} \times 25.4 \mathrm{~mm}$ cross section with large rounded corners and a modular length of up to $160 \mathrm{~mm}$, depending on measurement equipment and test case. The combustion chamber has a single shear-coaxial injector with an inner diameter of $1.2 \mathrm{~mm}$, an oxygen post width of $0.5 \mathrm{~mm}$, and a hydrogen annulus gap of $0.244 \mathrm{~mm}$. The supply of hydrogen and oxygen is pressure fed, with fluids at room temperature. There is an optical window next to the faceplate of the injector to enable measurements of $\mathrm{OH}$ concentration. The combustion chamber ends with a convergent-divergent supersonic nozzle which expands into ambient conditions. 
TABLE 3: Experimental conditions for the reacting ("combustion") test case [7].

\begin{tabular}{lccccc}
\hline $\begin{array}{l}\text { Pressure, } \\
\text { bar }\end{array}$ & $\begin{array}{c}\mathrm{O} / \mathrm{F}^{*} \\
\text { vel. ratio }\end{array}$ & $\begin{array}{c}\mathrm{H}_{2} \text { vel., } \\
\mathrm{m} / \mathrm{s}\end{array}$ & $\begin{array}{c}\mathrm{O} / \mathrm{F} \\
\text { mass ratio }\end{array}$ & $\begin{array}{c}\mathrm{H}_{2} \text { mass flux, } \\
\mathrm{g} / \mathrm{s}\end{array}$ & $\begin{array}{c}\text { Uncertainty, } \\
\%\end{array}$ \\
\hline 53 & 0.40 & 93.4 & 3.85 & 0.75 & 23 \\
\hline${ }^{*} \mathrm{O} / \mathrm{F}-$ oxidizer/fuel, that is, oxygen/hydrogen & & &
\end{tabular}

${ }^{*} \mathrm{O} / \mathrm{F}$-oxidizer/fuel, that is, oxygen/hydrogen.

The experiments were performed at combustion chamber pressures between 10 and 53 bar. The test case at high pressure is only present in the current paper (see Table 3), since it is the closest by pressure to real combustion chambers. The test duration was about 8 seconds due to heat release reasons. The measurements were taken in the last 1-2 seconds. The $\mathrm{OH}$ images were shot with a frequency of $10 \mathrm{~Hz}$, so the frame rate is orders of magnitude slower than the flow inside the combustion chamber. Therefore, nothing can be said about the temporal development of the flame, and the acquired pictures can only be regarded as a random set of the instantaneous images of the $\mathrm{OH}$ distribution. It is also worth pointing out that the uncertainty of the experimental conditions is relatively high and amounts to $23 \%$ in $\mathrm{OH}$ number density.

3.2. Simulations. The numerical model, which had been developed for the modeling of mixing, was extended to simulate the flame of the coaxial injector. In experiments [7], a broken flame front was observed. The broken flame front is an indicator for unsteady flow. The flame itself is the source of instability due to the large density gradient on the flame front. For this reason, reactive flows are unstable in most cases. The RANS equations are not suitable for the modeling of unstable flows. To model the unstable flow the ScaleAdaptive Simulation (SAS) turbulence model [17], which is an improved unsteady RANS (URANS) formulation, has been employed. The SST-SAS model is a two-equation turbulence model, which can be operated in URANS and LES-like modes depending on the von Karman length scale and the mesh resolution. The SAS formulation introduces an additional source term into the transport equation for the turbulence eddy frequency $\omega$, which is defined by the von Karman length scale. The SAS model has a LES-like behavior in unsteady regions of the flow field. In regions of stable flow, the SAS model switches to the SST turbulence model. To speed up the calculations, the stationary solution obtained with the help of RANS was used as the initial data in the subsequent transient simulations using the SAS model.

The combustion process has been modeled using the assumption of thin flame: chemical time-scale is smaller than the turbulent, transport, and flow timescales. The assumption of thin flame holds well in $\mathrm{GO}_{2}-\mathrm{GH}_{2}$ flames at high pressures which was shown in works $[18,19]$. The mixture composition is modeled using a flamelet approach [20]. The advantage of the flamelet approach is the possibility of evaluating the mass fraction of intermediate species such as $\mathrm{OH}$ or other radicals and therefore of performing the comparison with optical measurements, while no need arises to solve for additional species transport equations. To couple laminar flamelet with turbulence, the mixture fraction is integrated over the probability density function (PDF). The flamelet library was generated using built-in tool CFX-RIF [8]. The generation of the flamelet library was carried out using the default settings and the default hydrogen reaction mechanism with 9 species without $\mathrm{NO}_{x}$.

The flame propagation was modeled with the help of the burning velocity model (BVM), also called Turbulent Flame Closure (TFC). The BVM model is one of the builtin combustion models in CFX [8]. The BVM uses algebraic correlations for modeling the turbulent burning velocity (the propagation speed of the flame in turbulent flow). The Zimont correlation [21] has been used in the present work. To model the flame in pure oxygen, the value of $A$ was set to 1 , and the critical velocity gradient was increased to $10^{5} \mathrm{~s}^{-1}$ in the Zimont model. The parameters of the laminar flame, which are also required for the model, were calculated and modeled using hydrogen kinetic mechanism of Burke et al. [22] and computer code PREMIX [23], which is a part of the well-known software package CHEMKIN II [24].

A few words on transport properties need to be said because the flow becomes sensitive to them in the reacting test case. The same model of transport properties has been used in both test cases. The used model of transport properties was applied and described in more detail in recently published works $[3,10]$. The same values of the turbulent Prandtl and Schmidt numbers were used as before, namely, 0.9 and 0.7 , respectively. The molecular transport has been modeled using Sutherland's law with parameters recommended in [25]. The thermal conductivity and viscosity of the mixture were calculated using the Wassiljewa relation. (The usage of the Wassiljewa relation in the case of $\mathrm{H}_{2}-\mathrm{O}_{2}$ mixtures is discussed in detail in [26].)

Temperature of $\mathrm{H}_{2}-\mathrm{O}_{2}$ flames exceeds $3000 \mathrm{~K}$. At such temperature, it is necessary to take into account the fact that specific heat capacities of gases differ from the specific heat capacity of ideal gas. The temperature dependencies of heat capacities (also of enthalpies, and of entropies) of mixture components were calculated using NASA polynomials [27]. In CFX (and CFX-RIF) the coefficients of the polynomials are taken from the Chemkin Thermodynamic database [28].

A mesh convergence study had also been performed before the final simulations. The study gave similar results to the previous test case in terms of the flow field in injector post vicinity. 2D simulations were performed on mesh with 16 nodes on the tip of the oxidizer post. The $2 \mathrm{D}$ simulations showed that flame simulations require the $3 \mathrm{D}$ formulation. For this reason, 3D simulations were also performed. For them, a tetrahedral mesh was generated with a constant mesh density around the flame with 11 nodes on the tip of the oxidizer post. 

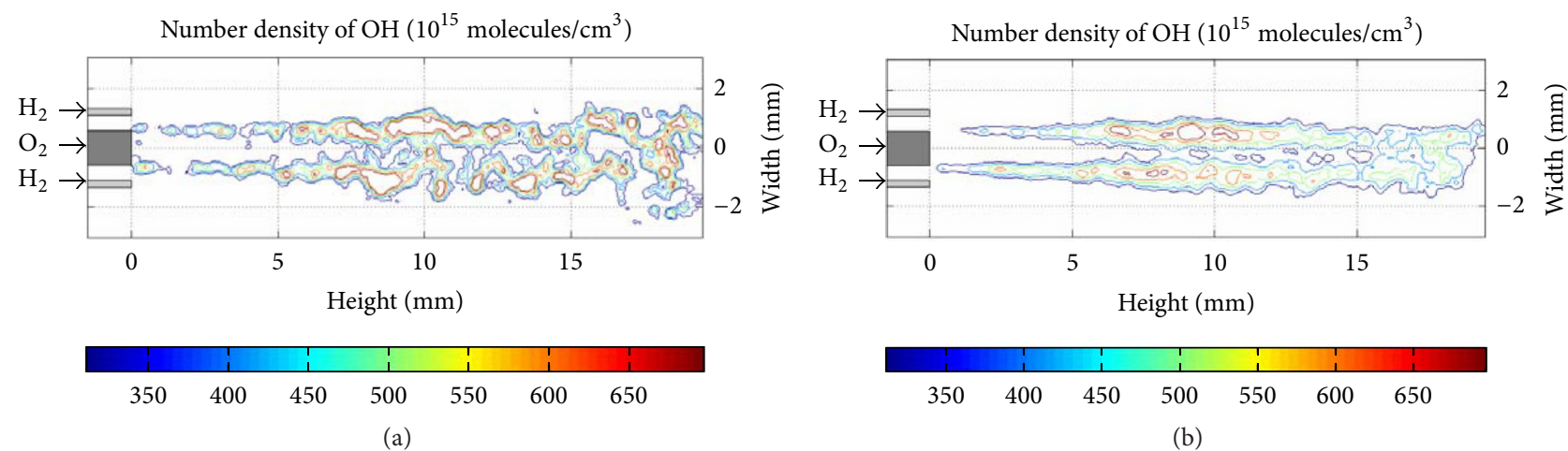

(a)

(b)

FIGURE 6: Instantaneous (a) and average (b) $\mathrm{OH}$ number density contours obtained by Vaidyanathan at pressure of 53 bar [7].

3.3. Results and Discussion. The performed simulations showed a new angle on the experimental data. Thirteen $\mathrm{OH}$ images were acquired at the rate of $10 \mathrm{~Hz}$ with $100 \mathrm{~ns}$ exposure time in experiments [7]. The results of $\mathrm{OH}$ measurements are shown in Figure 6. The advection time in the field of vision amounts to $20 \mathrm{~ms}$. Sozer [19] identified the local and global chemical timescales for this particular experiment. They both are orders of the magnitude larger than the exposure time. Therefore, all thirteen images are a random set of the instant shots. At the same time, the thirteen images are not enough to get the average $\mathrm{OH}$ image with good statistics in the present case. For this reason, the average $\mathrm{OH}$ image is corrupted by artifacts of separate instant $\mathrm{OH}$ shots, and this should be taken into account during the comparison with the simulation results, which do not suffer from these problems.

In the experiments [7], it was believed that the experimental data would be used for the quantitative comparison with the results of CFD simulations. According to calculations using NASA CEA [27], the equilibrium mole fraction of $\mathrm{OH}$ amounts at pressure of 53 bar to 0.11 in the stoichiometric hydrogen-oxygen mixture and to 0.0068 in the mixture with the equivalence ratio of 2.06 which corresponds to $\mathrm{OH}$ mole fractions in the flame front and the flame plume, respectively. These fractions correspond in turn to the $\mathrm{OH}$ concentrations of

$$
n_{\mathrm{OH}}=\frac{0.11 \cdot p}{k T} \approx 12000 \times 10^{15} \text { molecules } / \mathrm{cm}^{3}
$$

in the flame front, and $750 \times 10^{15}$ molecules $/ \mathrm{cm}^{3}$ in the flame plume. The measurements underestimate the $\mathrm{OH}$ concentration by order of magnitude in the flame; see Figure 6. Recently, Fiala showed that hydrogen-oxygen flames are optically thick at high pressures [29]. Vaidyanathan performed his calculations in the assumptions of optically thin media that unavoidably results in a lower concentration of $\mathrm{OH}$. There is also another issue which complicates an interpretation of the experiment. This is the small size of the window in the combustion chamber, which allows seeing only the first $20 \mathrm{~mm}$ of flame. With a larger window, it would be possible to clearly identify the flame length and to see $\mathrm{OH}$ concentration in the flame plume. The direct comparison between $\mathrm{OH}$ concentration in the plume and the estimation given by NASA
CEA [27] would be good criteria for cross-checking of the experimental method.

The results of simulations in the $2 \mathrm{D}$ axisymmetric domain are shown in Figure 7. The distinctive feature of the axisymmetric simulations is the large length of the flame due to the presence of the axis. The specific boundary conditions prevent closing of the flame at the axis of the domain. Due to the flame-turbulence interaction, the average $\mathrm{OH}$ mass fraction is significantly less than the $\mathrm{OH}$ mass fraction in the flame front. The flame holding occurs in the stagnate region behind the injector post. The pockets of the burnt gas arise in the wake of the injector tip and grow and travel further downstream. They pass approximately a half of the window within $0.5 \mathrm{~ms}$. The burnt gases separate the reactants and prevent their mixing. Therefore, the reactants need more time for the mixing compared to a nonreactive case. The presence of the layer of burnt gases, which separate the reactants, is a main distinctive feature of the current case from the previous (nonreactive) case. However, the combustion of fuel directly behind the injector post generates the vortex shedding. (Due to large difference in density of burnt and unburnt gases, the flame is a powerful source of instability.) The vortices traveling along the flame significantly speed up the mixing in the flame.

Although the experimental data do not allow quantitative comparison, the qualitative comparison is sensible. The $2 \mathrm{D}$ simulations visibly differ from the experimental observations in contrast to the $2 \mathrm{D}$ simulations in the previous test case. The two-dimensional axisymmetric problem settings render the formation of turbulence structures in the circumferential directions impossible. Under such conditions, the SAS model runs in the RANS mode and produces the same results as URANS simulations. The SAS model is formulated in such way that it runs in the RANS mode until the turbulence produces a sufficiently high inhomogeneity of flow even under the conditions of large eddy viscosity. (By this reason, the SAS model does not always switch into the LES-like mode without an external source of instability [30].) In the present case, the inhomogeneity of the flow is not enough for the transition into the LES-like mode.

In order to get more realistic results, three-dimensional (3D) simulations have been performed. The employed 3D 


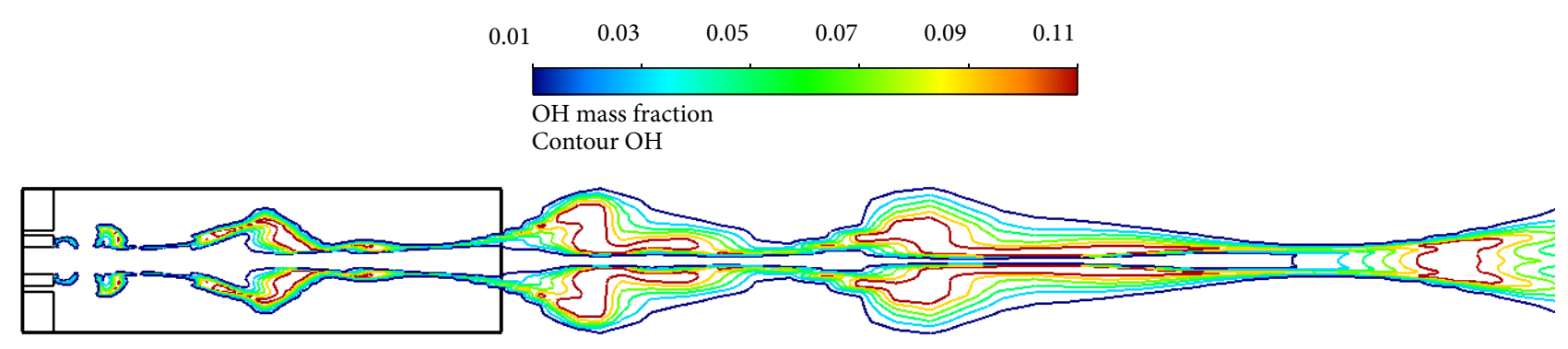

(a)

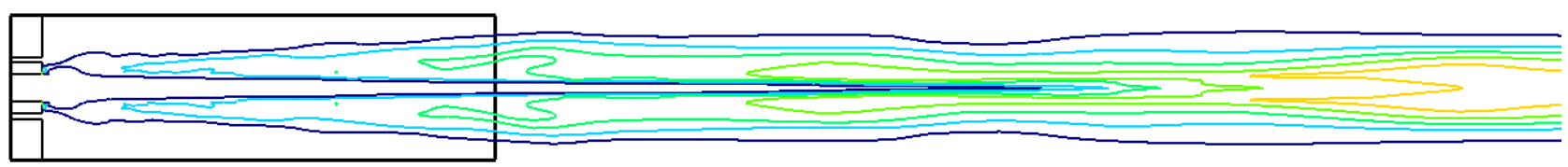

(m)

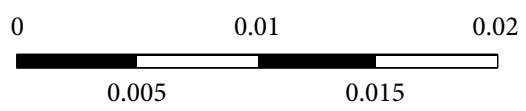

(b)

FIGURE 7: Instantaneous (a) and time-average (b) OH contours computed in 2D axisymmetric domain.

computational domain represents a half of the chamber dissected in the longitudinal direction. The $3 \mathrm{D}$ numerical model allows developing of turbulent structures in any direction; thus, there is a chance to get more realistic results. (In the previously used axisymmetric domain, the boundary conditions strongly limited the formation of eddies and the transverse motion of gas especially near the centerline.) The results of the $3 \mathrm{D}$ simulation are shown in Figure 8 . The simulating results are in a qualitative agreement with the experimental observations. The switch from the $2 \mathrm{D}$ computational domain to the $3 \mathrm{D}$ domain increased the computational time approximately 10 times.

In the $3 \mathrm{D}$ simulation, the frequency of the vortex shedding is higher than in the $2 \mathrm{D}$ simulations, and the shedded vortices are smaller. The flame length is practically twice shorter than in the 2D simulations; see Figure 8 . In the presence of additional degrees of freedom, the oxygen jet dissipates faster. In contrast to the $2 \mathrm{D}$ simulations, here we can see the evolution of eddies. Moving downstream they grow and simultaneously dissipate. One can see that the flame is wider in the simulations than in reality; see Figure 6. It should be borne in mind that the SAS model is not an equal substitute of LES. In the present simulations, the eddy viscosity is about ten times larger than the molecular viscosity. Therefore, the mechanism of eddy growth and dissipation is different from the existing one in the nature.

In spite of the similarity, the test cases are characterized by the different flow fields and therefore have been modeled by different turbulence models. In contrast to the (first) nonreacting test case, the reacting test case is conditioned by the presence of an $\mathrm{H}_{2} \mathrm{O}$ layer which separate fuel and oxidizer. The second test case is characterized by a strong interplay of two processes: the formation of the $\mathrm{H}_{2} \mathrm{O}$ layer and the onset of the strong Kelvin-Helmholtz instability occurring due to the large density and velocity gradients in the $\mathrm{H}_{2} \mathrm{O}$ layer. The first process slows down the combustion of the propellants while the second speeds it up. That is why it is critical to simulate accurately the evolution of the layer of the burnt gases and therefore to employ the scale-resolved turbulence models (i.e., SAS, DES, LES, etc.) in the second test case.

In the first test case [6], the Kelvin-Helmholtz instability was not observed because the density gradient was 100 times smaller. The application of the numerical model, which was used successfully in the nonreacting test case, resulted in the flame that was longer than the numerical domain.

\section{Conclusions}

The mixing and combustion of the propellants injected by a single shear-coaxial injector have been simulated using the CFD code ANSYS CFX. The numerical results have been compared with the results of the experimental observations.

The mixing in the jet of a coaxial injector has been modeled using the stationary SST turbulence model in the 2D axisymmetric numerical domain. The numerical results agree very well with the experimental data on propellant mixing.

The grid convergence study showed that the numerical grid needs 8-15 nodes at the injector tip to form the recirculation zone over the tip and 15-30 nodes to get a mesh independent solution.

To simulate the flame, the numerical model was extended by the BVM combustion model and the unsteady SAS-SST turbulence model. In contrast to the nonreacting test case, 


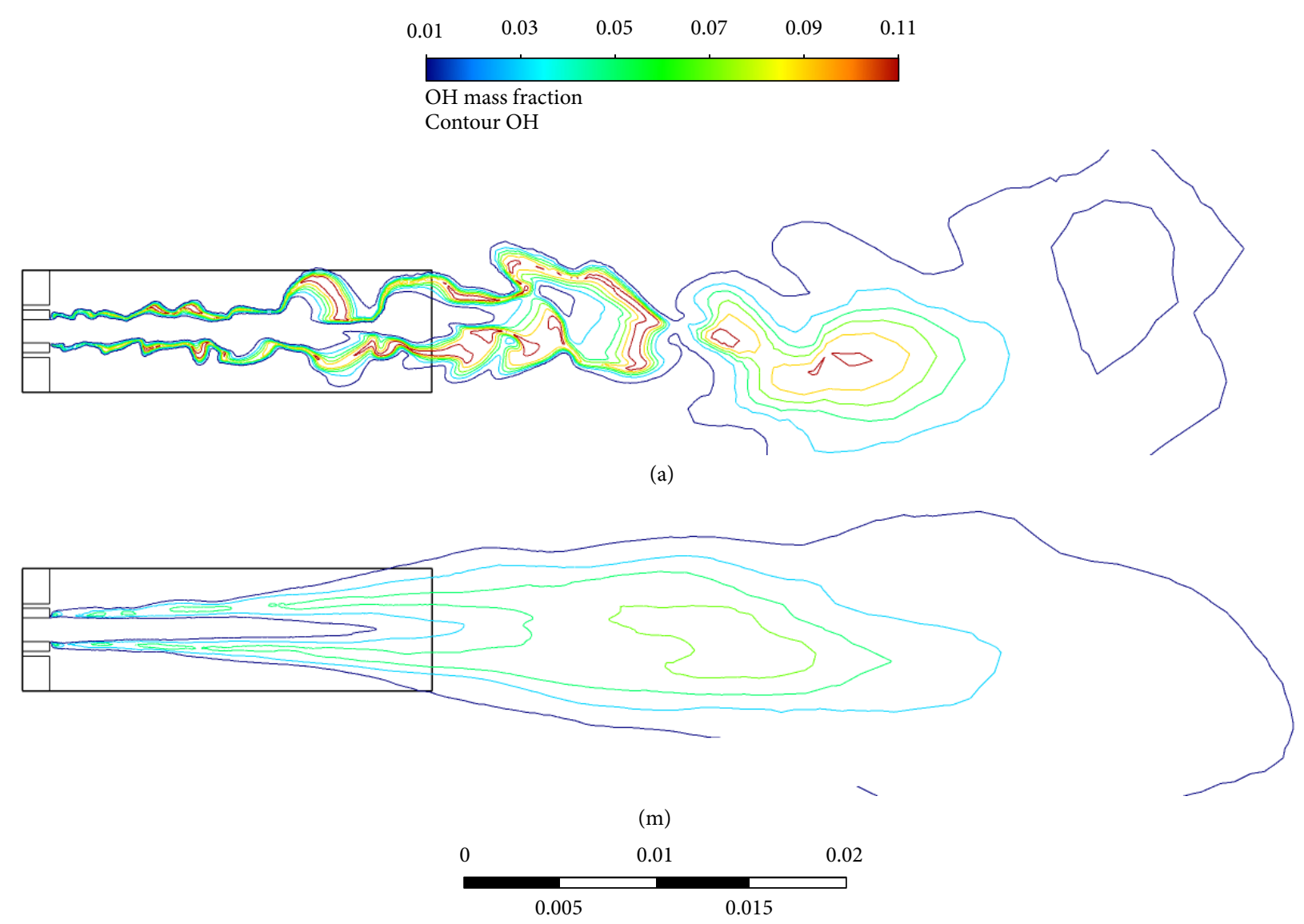

(b)

FIgURE 8: Instantaneous (a) and time-average (b) $\mathrm{OH}$ contours computed in 3D domain.

the experimental results by Vaidyanathan [7] do not allow performing the quantitative comparison with the numerical results. However, on the qualitative level, the results obtained with the SAS model agree with experimental observations.

The flame of a coaxial injector is held in the stagnation region behind the injector tip. In the flame, the propellants are separated by the layer of burnt gases. The numerical model, which agrees well with the experiment in the simulations of the flow of nonreacting gases, results in an extremely large flame. The flame becomes reasonably short and qualitatively similar to the observed flame in the experiment after switching to the SAS turbulence model and the three-dimensional numerical domain. However, the developed 3D numerical model still can be improved because it resolves only some turbulent scales, what one can see comparing the simulations with the experiment.

The comparison of the studied test cases emphasizes the complexity of flame simulations. The layer of hot gases produced in chemical reactions results in the onset of the Kelvin-Helmholtz instability. Hence, the CFD simulations of the flame of coaxial injectors require the use of scale-resolved turbulence models.

\section{Disclosure}

The present address of Markus Feil is as follows: Institute of Aircraft Propulsion Systems, University of Stuttgart, Pfaffenwaldring 6, 70569 Stuttgart, Germany. The results of this work were presented at the 5 th European Conference for Aerospace Sciences in Munich, Germany, July 2013.

\section{Conflicts of Interest}

The authors declare that there are no conflicts of interest regarding the publication of this paper.

\section{References}

[1] P. K. Tucker, S. Menon, J. C. Oefelein, V. Yang, and C. L. Merkle, "Validation of high-fidelity CFD simulations for rocket injector design," in Proceedings of 44th AIAA/ASME/SAE/ASEE Joint Propulsion Conference and Exhibit, USA, July 2008.

[2] S. Pal, W. Marshall, R. Woodward, and R. J. Santoro, "Wall heat flux measurements for a uni-element GO2/GH2 shear coaxial injector," in Proceedings of 3rd International Workshop "Rocket Combustion Modeling", Snecma, Safran Group, Vernon, France, 2006. 
[3] V. P. Zhukov, "Computational fluid dynamics simulations of a GO2/GH2 single element combustor," Journal of Propulsion and Power, vol. 31, no. 6, pp. 1707-1714, 2015.

[4] J. Oefelein, "Mixing and combustion of cryogenic oxygenhydrogen shear-coaxial jet flames at supercritical pressure," Combustion Science and Technology, vol. 178, no. 1-3, pp. 229$252,2006$.

[5] L. Vingert, A. Nicole, and M. Habiballah, "The Mascotte single injector 60 bar hot test for code validation-test-case specifications and some more experimental data," in Proceedings of in 3rd International Workshop Rocket Combustion Modeling, Snecma, Safran Group, Vernon, France, 2006.

[6] S. Schumaker, An experimental investigation of reacting and nonreacting coaxial jet mixing in a laboratory rocket engine $[\mathrm{PhD}$. thesis], University of Michigan, USA, 2009.

[7] A. Vaidyanathan, OH-PLIF measurements and accuracy investigation in high pressure GH2/GO2 combustion [PhD. thesis], University of Florida, USA, 2008.

[8] ANSYS Inc., ANSYS CFX-Solver Theory Guide, Release 13.0, Canonsburg, PA, USA, November 2010.

[9] I. Yimer, I. Campbell, and L.-Y. Jiang, "Estimation of the turbulent Schmidt number from experimental profiles of axial velocity and concentration for high-Reynolds-number jet flows," Canadian Aeronautics and Space Journal, vol. 48, no. 3, pp. 195200, 2002.

[10] V. P. Zhukov and D. I. Suslov, "Measurements and modelling of wall heat fluxes in rocket combustion chamber with porous injector head," Aerospace Science and Technology, vol. 48, pp. 6774, 2016.

[11] O. Knab and D. Preclik, "Test case RCM-3 EADS-ST subscale chamber," in Proceedings of the 3rd International Workshop "Rocket Combustion Modeling", Snecma, Safran Group, Vernon, France, 2006.

[12] B. Mewes, M. Frey, J. Görgen, and O. Knab, "Simulations of the EADS-ST subscale combustor using a dense gas approach," in Proceedings of 3rd International Workshop "Rocket Combustion Modeling”, Snecma, Safran Group, Vernon, France, 2006.

[13] L. Ballester, S. Zurbach, and C. Pauly, "3D simulations of the EADS-ST subscale chamber incorporating real gas effects," in Proceedings of 3rd International Workshop "Rocket Combustion Modeling”, Snecma, Safran Group, Vernon, France, 2006.

[14] D. Wennerberg, M. Frey, J. Görgen, and O. Knab, "3D spray combustion modeling of the EADS-ST subscale combustor," in Proceedings of 3-rd International Workshop "Rocket Combustion Modeling”, Snecma, Safran Group, Vernon, France, 2006.

[15] R. Langtry, A Correlation-Based Transition Model Using Local Variables for Unstructured Parallelized CFD codes, University of Stuttgart, Germany, 2006.

[16] D. Davis and B. Chehroudi, "Measurements in an acoustically driven coaxial jet under sub-, near-, and supercritical conditions," Journal of Propulsion and Power, vol. 23, no. 2, pp. 364374, 2007.

[17] F. R. Menter and Y. Egorov, "The scale-adaptive simulation method for unsteady turbulent flow predictions. part 1: theory and model description," Flow, Turbulence and Combustion, vol. 85, no. 1, pp. 113-138, 2010.

[18] B. Ivancic and W. Mayer, "Time-and length scales of combustion in liquid rocket thrust chambers," Journal of Propulsion and Power, vol. 18, no. 2, pp. 247-253, 2002.

[19] E. Sozer, Modeling of Gaseous Reacting Flow and Thermal Environment of Liquid Rocket Injectors, University of Michigan, USA, 2010.
[20] N. Peters, Turbulent Combustion, Cambridge Monographs on Mechanics, Cambridge University Press, Cambridge, UK, 2000.

[21] V. Zimont, "Gas premixed combustion at high turbulence. Turbulent flame closure combustion model," Experimental Thermal and Fluid Science, vol. 21, no. 1-3, pp. 179-186, 2000.

[22] M. P. Burke, M. Chaos, Y. Ju, F. L. Dryer, and S. J. Klippenstein, "Comprehensive H2/O2 kinetic model for high-pressure combustion," International Journal of Chemical Kinetics, vol. 44, no. 7, pp. 444-474, 2012.

[23] R. J. Kee, J. F. Grcar, M. D. Smooke, and J. A. Miller, "A Fortran program for modeling steady laminar one-dimensional premixed flames," SAND85-8240, Sandia National Laboratories, Livermore, CA, USA, 1985.

[24] R. Kee, F. Rupley, and J. Miller, "CHEMKIN-III: A Fortran chemical kinetics package for the analysis of gas-phase chemical and plasma kinetics," Tech. Rep. SAND-96-8216, 1996.

[25] F. M. White, "Preliminary concepts", in Viscous Fluid Flow, pp. 29-32, McGraw-Hill, NY, USA, 2 edition, 1991.

[26] V. P. Zhukov and M. Pätz, "On thermal conductivity of gas mixtures containing hydrogen," Heat and Mass Transfer, vol. 53, no. 6, pp. 2219-2222, 2017.

[27] B. J. McBride and S. Gordon, "Computer program for calculation of complex equilibrium compositions and applications," Tech. Rep. 1311, NASA, 1996.

[28] R. J. Kee, F. M. Rupley, and J. A. Miller, "The Chemkin thermodynamic database," SAND87-8215, Sandia National Laboratories, Livermore, CA, USA, 1987.

[29] T. Fiala, Radiation from high pressure hydrogen-oxygen flames and its use in assessing rocket combustion instability [PhD. thesis], Technische Universität München, Germany, 2015.

[30] F. Menter, "Best practice: Scale-resolving simulations in ANSYS CFD. version 1.0," ANSYS Germany GmbH, 2012. 


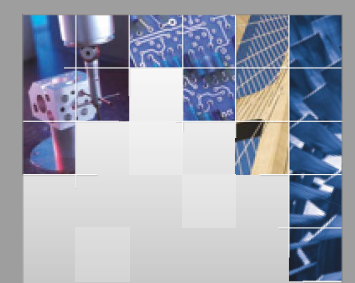

\section{Enfincering}
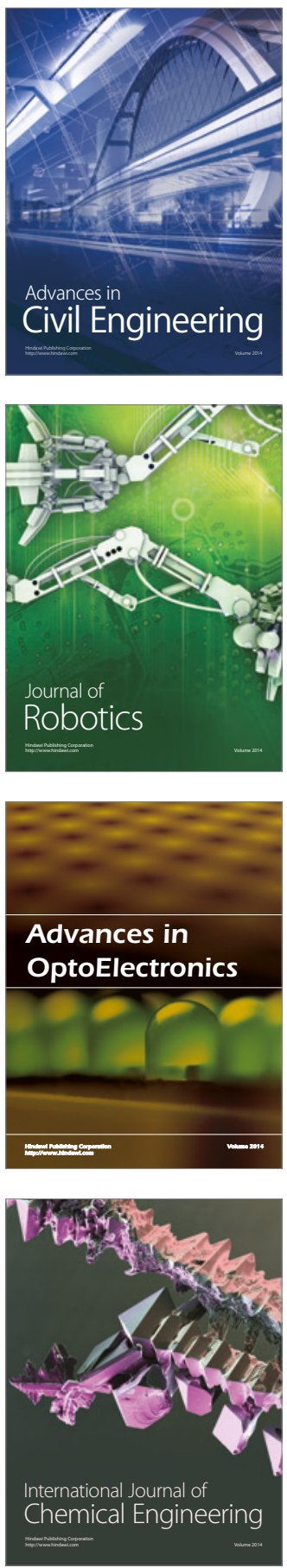

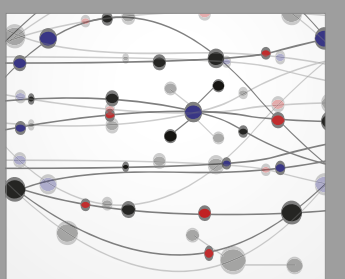

The Scientific World Journal

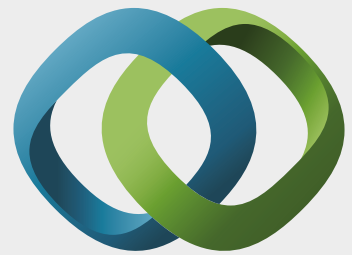

\section{Hindawi}

Submit your manuscripts at

https://www.hindawi.com
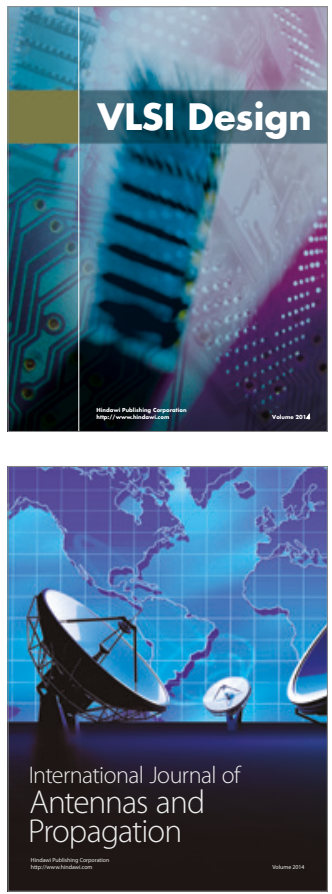

\section{Rotating}

Machinery
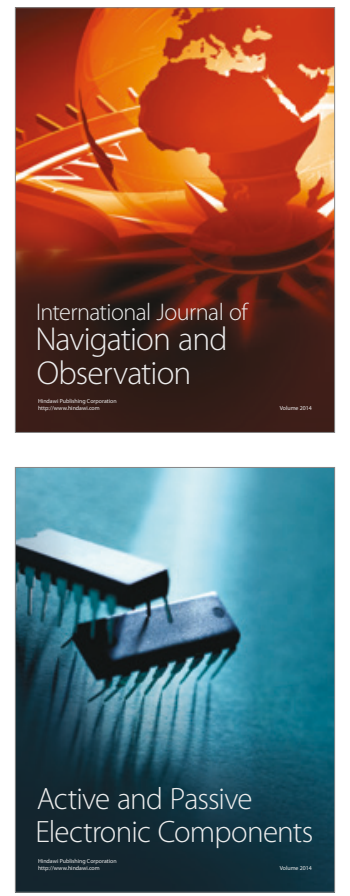
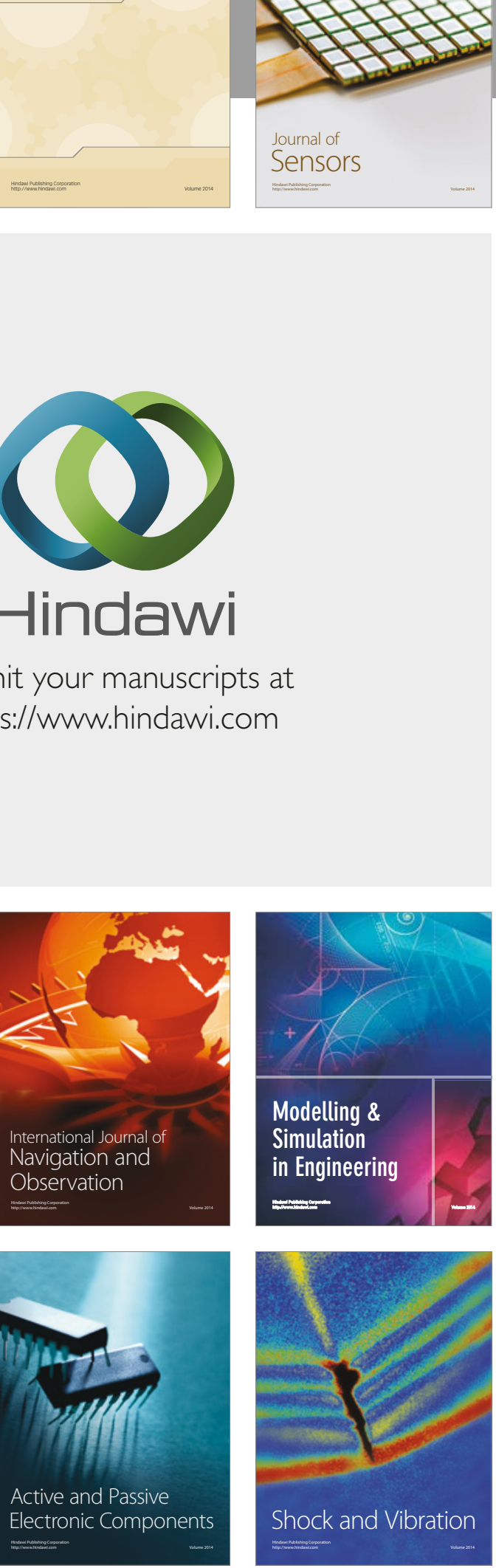
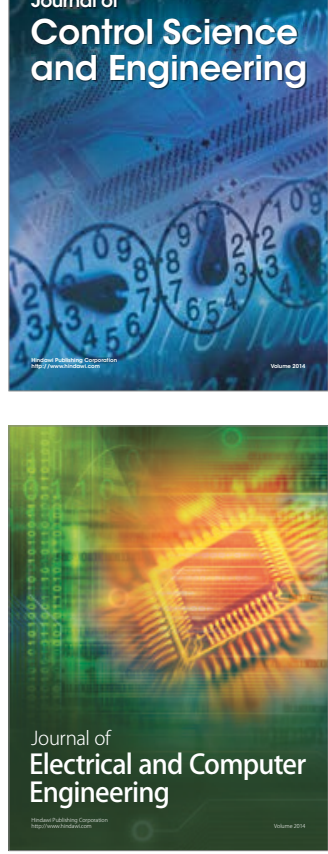

Distributed

Journal of

Control Science

and Engineering
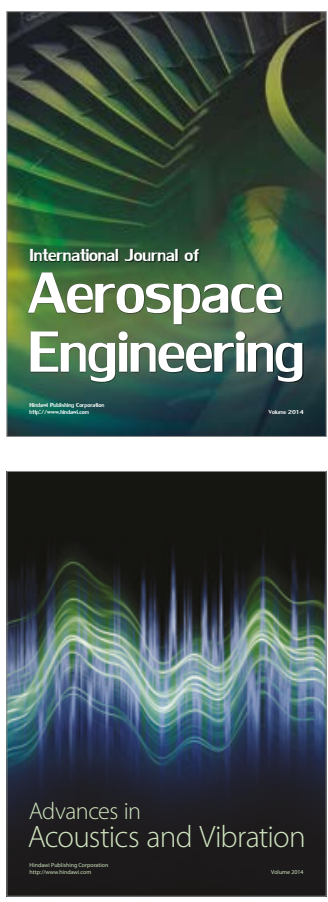

Sensor Networks 